\title{
Questes
}

\section{Héloïse et Abélard. Amants, époux, religieux}

\section{Fanny Oudin}

\section{(2) OpenEdition}

\section{Journals}

Édition électronique

URL : https://journals.openedition.org/questes/2380

DOI : 10.4000 /questes.2380

ISSN : 2109-9472

\section{Éditeur}

Les Amis de Questes

\section{Édition imprimée}

Date de publication : 15 février 2011

Pagination : 38-53

ISSN : 2102-7188

\section{Référence électronique}

Fanny Oudin, « Héloïse et Abélard. Amants, époux, religieux », Questes [En ligne], 20 | 2011, mis en ligne le 01 janvier 2014, consulté le 26 août 2021. URL : http://journals.openedition.org/questes/2380 ;

DOI : https://doi.org/10.4000/questes.2380 


\title{
Héloïse et Abélard. Amants, époux, religieux
}

\author{
Fanny OUDIN
}

Évoquer le couple formé par Héloïse et Abélard à propos des relations entre maris et femmes peut paraître surprenant, puisque tous deux sont d'ordinaire plutôt inscrits au nombre des amants célèbres. Ces deux esprits brillants, épris de philosophie, ont pourtant été successivement maître et élève, amants, époux pour complaire à Fulbert, moine et moniale à la suite de la castration d'Abélard, abbé et abbesse après la fondation de l'abbaye du Paraclet ${ }^{1}$. Parmi ces multiples figures, trois surtout ont retenu

\footnotetext{
${ }^{1}$ Les différents moments de la vie d'Abélard et Héloïse sont connus principalement d'après le tableau qu'en dresse Abélard lui-même dans l'Historia Calamitatum, une lettre de consolation adressée à un ami anonyme. Abélard y retrace son ascension intellectuelle à travers les controverses avec ses maîtres, puis ses amours avec Héloïse, sa castration, leur conversion et leur installation, pour l'un à Saint-Denis, pour l'autre à Argenteuil, la condamnation de sa Théologie au Concile de Soissons, ses démêlés avec les moines de Saint-Denis, son installation près de Troyes où il fonde un oratoire dédié au Saint-Esprit, le Paraclet, son départ pour l'abbaye de Saint-Gildas, près de Vannes, dont il a été élu abbé mais où il se heurte rapidement à l'hostilité de ses moines, et l'installation d'Héloïse et de ses sœurs, expulsées de l'abbaye d'Argenteuil, au Paraclet. Ce récit autobiographique est la première pièce du dossier de huit lettres qui forme la Correspondance d'Abélard et Héloïse. Celle-ci commence véritablement avec la Supplique (Deprecatoria) qu'Héloïse adresse à Abélard en réaction à l'Historia Calamitatum, qu'elle vient de lire. Elle reproche à Abélard de l'oublier, elle, son amante et son épouse, qui lui a toujours tout sacrifié et a toujours obéi à ses ordres, en se mariant malgré ses réticences puis en se faisant religieuse sans en avoir la vocation, et d'oublier les religieuses du Paraclet, qui sont, comme elle, ses filles spirituelles. Elle le supplie de leur écrire pour leur donner de ses nouvelles et les soutenir dans leur vie de moniales par des lettres de direction. Insatisfaite de la réponse d'Abélard, qui l'incite à prier pour son salut à l'exemple des saintes femmes qui ont su obtenir la grâce divine par leurs prières pour leurs proches, elle le relance par une lettre de lamentations. Après lui avoir reproché la démarche adoptée dans sa réponse, elle s'insurge contre l'acharnement de Fortune sur eux et l'injustice divine manifestée par la castration d'Abélard. Elle conclut sur un autoportrait en hypocrite, menant pour l'extérieur une vie de continence religieuse, mais continuant de regretter intérieurement le péché de ses amours passées. À cette lettre, Abélard répond en quatre points, en s'appuyant sur un commentaire du Cantique des cantiques pour prouver à Héloïse qu'épouse du Christ, elle a la précellence sur lui, simple serviteur de Dieu, et que seul son nouvel époux, qui a enduré la plus ignominieuse des passions pour racheter son âme, peut lui rendre
} 
l'attention. Pour Pierre le Vénérable ${ }^{2}$, Héloïse est une femme douée d'une science et d'une sagesse étonnantes, et la compagne aimante d'un grand philosophe devenu à la fin de sa vie un modèle de vertu monastique. Cette unité de la personnalité paraît se disloquer à l'époque moderne : l'amante passionnée s'oppose, pour certains, à la sage abbesse. De l'épouse, il n'est jamais question, comme si ce statut devait ternir l'éclat de la passion. Pourtant, chez Jean de Meun ${ }^{3}$, le mariage joue un rôle important dans la constitution de la figure d'Héloïse, ne serait-ce que par le contexte de son apparition, le discours qu'Ami fait tenir au mari jaloux. Sa vertu paraît exceptionnelle par contraste avec les mauvaises épouses. Mais la grandeur

l'amour pur et désintéressé qu'elle éprouve. Plutôt que de s'insurger contre Dieu, elle doit lui rendre grâce, et pleurer sur ses souffrances au lieu de plaindre celles d'Abélard, qui étaient méritées et se sont révélées un don de la grâce divine : action d'un bon médecin, action du père corrigeant son enfant plutôt que d'un ennemi cherchant sa mort. Ainsi, elle doit donc prier son nouvel époux pour son propre salut et celui de son ancien époux. À ces objurgations, Héloïse répond par une obéissance stricte : elle promet le silence sur ses anciennes plaintes, et propose de remplacer ce discours par un autre plus édifiant, en demandant à Abélard un traité sur l'origine des ordres religieux féminins et une nouvelle règle pour l'abbaye du Paraclet. Cette dernière demande est particulièrement développée, à travers des remarques qui pointent l'inadéquation de la Règle de saint Benoît à la condition féminine et la nécessité de l'adapter selon des principes qui mettent en valeur non la continence extérieure, faite pour le jugement des hommes, mais la pureté intérieure, qui seule importe à Dieu. Les deux dernières lettres constituent la réponse d'Abélard à ces deux demandes, avec d'abord un traité sur l'origine des ordres religieux, puis une Règle, dont l'authenticité a fait débat parmi les spécialistes de la correspondance. Cet échange qui forme en propre, dans les manuscrits, la correspondance d'Abélard et Héloïse a été suivi d'autres envois, tous centrés sur la vie spirituelle du Paraclet. Outre un recueil d'Hymnes et un autre de Sermons, ont été conservées une lettre-traité intitulée De Studio Litterarum et une Expositio in Hexaemeron. S'y ajoutent les Problemata Heloisae, un recueil de quarante-deux questions liées aux difficultés rencontrées par Héloïse et ses sœurs lors de leur lecture des textes religieux, avec les réponses données par Abélard, et la Confessio fidei ad Heloisam, écrite en 1139, au moment du Concile de Sens parallèlement à la confession plus générale, adressée à l'ensemble de l'Église, exigée de lui par le Concile.

${ }^{2}$ Dans la lettre de consolation qu'il adresse à Héloïse à la mort d'Abélard. Cf. The Letters of Peter the Venerable, Giles CONSTABLE (éd.), London, Harvard University Press, 1967, vol. 1, p. 303-308.

${ }^{3}$ Qui est à la fois le premier traducteur de la Correspondance en français et le premier à avoir véritablement mis en valeur la figure de l'amante comme telle. 
de la femme, à la fois comme amie et comme intellectuelle, se démontre précisément par son refus du mariage :

Pierre Abaalars reconfesse

Que suer Heloÿs, abbaesse

Dou Paraclet, qui fu s'amie,

Accorder ne se voloie mie

Pour riens qu'il la preïst a fame ;

Ainz li faisoit la juene dame

Bien entendant et bien lettrée

Et bien amant et bien amée,

Argumenz a lui chastier

Qu'il se gardast de marier. ${ }^{4}$

Ces quelques vers qui introduisent l'exemple dans le Roman de la Rose suffisent à montrer par la disposition des rimes que trois figures d'Héloïse, et non deux, sont en jeu, l'amante, l'abbesse et l'épouse. Les connaissances d'Héloïse réunissent les figures de l'abbesse et de l'amie ${ }^{5}$, dont l'amour se nourrit de sa culture et naît au contact des livres, comme le suggère le parallèle des vers 8767-8768. En revanche cette «letreüre » lui permet de « vaintre et donter»sa « nature » féminine, liée au statut d'épouse, ce qui oppose cette dernière figure aux deux autres.

Comme il le signale lui-même, Jean de Meun s'inspire à la fois de l'Historia Calamitatum, où Abélard retranscrit les arguments d'Héloïse contre le mariage au moment où celui-ci est conclu, et du passage de la supplique d'Héloïse où celle-ci, complétant le récit de son époux, ajoute un argument supplémentaire selon lequel elle préférerait «Estre ta putain

4 Guillaume De Lorris ET JeAn DE Meun, Le Roman de la Rose, Armand Strubel (éd.), Paris, Livre de Poche, «Lettres Gothiques », 1992, v. 8763-8772 (je souligne). Il faudrait, pour plus de clarté, citer l'ensemble de l'exemple qui se poursuit jusqu'au vers 8834 et en analyser toutes les nuances, en particulier dans son effort pour caractériser ce que pouvait avoir de singulier le savoir d'Héloïse comme femme, qui met en jeu une opposition intéressante entre «nature» et «lettreüre ». Au-delà du plaidoyer en faveur de l'amour libre, les vers de Jean de Meun témoignent d'une grande sensibilité à la complexité des rapports entre Abélard et Héloïse : malgré la constitution d'Héloïse en exemple mis au service d'une thèse, il ne sépare pas plus que Pierre le Vénérable les différents aspects de sa personnalité.

${ }^{5} \mathrm{Au}$ lieu de les opposer comme dans la controverse moderne. 
apelée / Qu'empereriz coronnée ${ }^{6}$. La reprise d'une ancienne argumentation, environ quinze ans après les faits, alors même que ce mariage n'est plus d'actualité puisqu'il a été légalement rompu par leur commune entrée dans les ordres, suggère que le mariage a joué un rôle plus important dans la définition de l'identité du couple que ne l'aurait laissé supposer la vision moderne d'Héloïse. Le comprendre suppose d'abord une analyse précise des arguments contre le mariage tels qu'ils ont été effectivement formulés par Abélard et Héloïse. Cela implique aussi de se demander quel est l'ethos auquel tous deux recourent, comme correspondants, lorsqu'ils définissent la relation sur laquelle se fonde leur échange épistolaire. Les salutations et les noms qu'ils se donnent peuvent indiquer si leurs lettres se veulent épîtres d'amants, d'époux, ou de religieux $^{7}$ : leur correspondance pourrait alors fournir un point de référence pour déterminer si, au Moyen Âge, une correspondance entre époux peut accéder au statut de texte littéraire, si le discours que s'adressent deux époux peut avoir la valeur d'une parole poétique.

L'argumentation d'Héloïse contre le mariage dans l'Historia Calamitatum repose principalement sur l'opposition de l'état d'époux avec le statut de clerc et de philosophe qui est celui d'Abélard. Comme l'a montré Étienne Gilson, cette incompatibilité ne tient pas à des dispositions légales mais plutôt à un idéal commun de gloire philosophique jugé peu conciliable avec la vie d' «âne domestique » enchaîné à une femme par un

\footnotetext{
${ }^{6}$ Guillaume de LoRris et JeAn De Meun, Le Roman de la Rose, éd. cit., v. 88278828.

${ }^{7}$ Les problèmes que soulève le jeu sur les salutations au sein de la correspondance sont cependant trop importants pour pouvoir être traités intégralement dans cet article, qui se bornera donc aux remarques nécessaires pour la question du mariage, sans prétendre régler en même temps l'épineuse question de la conversion d'Héloïse.
} 
rapport de servitude mutuelle ${ }^{8}$. Lorsqu'il reprend cette opposition dans la lettre V, non plus pour sa propre gloire mais pour celle d'Héloïse, Abélard met en valeur l'antagonisme des fonctions intellectuelles et des fonctions purement reproductrices, en opposant la génération physique à l'engendrement de «filles spirituelles». Ce qui sous-tend le rejet du mariage est donc d'abord la conscience de leur statut de lettrés et de philosophes, plus compatible avec le moniage qu'avec le mariage. En revanche, quoiqu'Abélard se peigne dans l'Historia en «recreant» momentanément détourné de la philosophie par l'amour, il n'en conclut pas pour autant à une incompatibilité entre l'amour et les lettres. L'amour ne tarit pas l'esprit d'Abélard, il le fait changer d'objet en transformant les leçons en chansons. Dans sa stratégie de séduction, Héloïse lui apparaît comme l'amante idéale précisément parce qu'ils pourront échanger des lettres. C'est le mariage, et lui seul, qui est considéré comme incompatible avec le travail de la pensée et avec l'écriture poétique. Il est possible que cette exclusion repose sur le schéma des trois fonctions de la culture indoeuropéenne : comme materfamilias ${ }^{9}$, l'épouse incarne, plus que l'amante, la fonction économique et reproductrice, et s'oppose donc en ce sens à la première fonction, celle des clercs, liée à l'écriture. Cela expliquerait que, quoique des correspondances entre époux aient été conservées par les archives médiévales, ce soit toujours au sein de collections plus vastes réunissant les lettres et les documents d'affaires de familles ${ }^{10}$, sans qu'une valeur poétique paraisse leur avoir été accordée.

\footnotetext{
${ }^{8}$ Dans Héloïse et Abélard, $3^{\mathrm{e}}$ édition revue, Paris, Vrin, 1978, p. 25-54. L'expression d' «âne domestique » est empruntée par Étienne Gilson au sermon XXXIII d'Abélard, sur Jean-Baptiste. Elle résume bien la description du mariage proposée par Héloïse dans l'Historia ou par Abélard dans la lettre V.

${ }^{9}$ Le terme se trouve dans la lettre VI d'Héloïse.

${ }^{10}$ On peut penser, par exemple, aux lettres de Margherita Datini, conservées au sein des archives de son époux, Francesco Datini, ou bien, en Angleterre, à celle des femmes des familles Paxton ou Stonor, pour ne citer que les plus connues.
} 
À ces considérations communes, Héloïse ajoute le refus de la contrainte : elle revient sur cet argument dans sa supplique en affirmant que

si uxoris nomen sanctius ac validius videtur, dulcius michi semper extitit amice vocabulum, aut - si non indigneris concubine vel scorti; ut, quo me videlicet pro te amplius humiliarem, ampliorem apud te consequerer gratiam [...] si me Augustus universo presidens mundo matrimonii honore dignaretur, totumque michi orbem confirmaret in perpetuo possidendum, karius michi et dignius videretur tua dici meretrix quam illius imperatrix. ${ }^{11}$

Héloïse donne donc deux formulations différentes de ce qui est apparemment la même idée. La seconde, qui clôt le paragraphe, crée dans le suivant une situation de mundus inversus, où les épouses qui se marient pour devenir « imperatrix » sont les véritables $«$ meretrix $»^{12}$ :

Nec se minime venalem estimet esse que libentius ditiori quam pauperi nubit et plus in marito sua quam ipsum concupiscit. Certe quamcumque ad nuptias hec concupiscentia ducit, merces ei potius quam gratia debetur. $^{13}$

Avec cette formule, Héloïse revient au thème introducteur :

Nichil umquam - Deus scit ! - in te nisi te requisivi ; te pure non tua concuspicens. Non matrimonii federa, non dotes

\footnotetext{
11 Toutes les citations latines sont tirées de l'édition donnée par Eric HICKS de la correspondance et de sa traduction en ancien français dans La Vie et les epistres Pierres Abaelart et Heloys sa fame, Paris, Honoré Champion, 1991 (lettre II, p. 49-50 pour cette citation et les suivantes). Les traductions données en note viennent de l'édition d'Eric HICKS et Thérèse MoReau, Lettres d'Abélard et Hélö̈se, Livre de Poche, "Lettres Gothiques », 2007. "Si le nom d'épouse peut sembler plus sacré et plus convenable, celui d'amante m'a toujours paru plus doux, ou même - si je ne craignais pas de t'offenser - celui de maîtresse ou de chienne. Car il me semblait que plus je m'abaissais, plus je méritais ta faveur [...] si le maître de l'univers, Auguste lui-même, avait voulu m'honorer en me prenant pour épouse et en me donnant la jouissance perpétuelle des trésors de la terre, j'aurais trouvé plus doux et plus digne qu'on me dise ta catin plutôt que son impératrice!», p. 145.

${ }^{12}$ Est-ce un hasard si les deux termes choisis par Héloïse riment?

${ }^{13}$ «Que celle qui préfère le mariage avec un riche plutôt qu'avec un pauvre, et qui brûle pour les biens du mari plutôt que pour lui-même, ose dire qu'elle ne se prostitue pas ! La femme poussée au mariage par ce genre de désir mérite plutôt compensation que reconnaissance. », éd. cit., p. 145.
} 
aliquas expectavi, non denique meas voluptates aut voluntates, sed tuas, sicut ipse nosti, adimplere studui. ${ }^{14}$

Mais elle dévie rapidement vers une nouvelle idée lorsqu'elle introduit comme figure d'autorité la philosophe Aspasie. Celle-ci est supposée avoir développé la même dénonciation des épouses vénales et le même éloge de l'amour pur et désintéressé comme fondement de toutes les relations entre hommes et femmes, mariage compris. Mais le propos prêté à la philosophe est finalement celui-ci :

Quare nisi hoc peregeritis, ut neque vir melior, neque femina in terris lectior sit, profecto semper id quod optimum putabitis esse multo maxime requiretis, ut et tu maritus sis quam optime, et hec quam optimo viro nupta sit. ${ }^{15}$

Héloïse le commente de la sorte :

Sancta profecto hec et plusquam philosophica est sententia ipsius sophie quam philosophie dicenda; sanctus hic error, et beata fallatia in conjugatis, ut perfecta dilectio illesa custodiat matrimonii federa, non tam corporum continentia quam animorum pudicitia. ${ }^{16}$

L'apparition du terme «dilectio » à la fin du passage implique que la préférence d'Héloïse pour le nom de «meretrix » ne repose pas sur l'idée que le mariage exclurait l'amour : au contraire, le mariage idéal se fonde selon elle sur une amitié «parfaite » entre les époux. Cette perfection, telle qu'Héloïse la définit, est de même nature que celle de l'amour en dehors du

\footnotetext{
14 «Dieu sait que je m'intéressais uniquement à ta personne et non à tes biens, que c'est toi que je désirais et uniquement toi. Je n'ai jamais attendu de toi, tu le sais bien, ni contrat de mariage ni prix de la fiancée. En fin de compte, je n'ai jamais cherché à satisfaire ni mes désirs ni mes envies, mais uniquement à te contenter. », ibid., p. 145.

15 «Ainsi donc, si vous ne réussissez pas à vous convaincre qu'il n'existe pas de meilleur mari ou de femme plus désirable sur terre, vous serez sans cesse en quête d'un plus grand bien, afin d'être, toi, le mari de la meilleure, et toi, l'épouse du meilleur des maris. », ibid., p. 147.

${ }^{16}$ «Ce conseil bienheureux est parfaitement juste ; il relève, il faut le dire, davantage de la sagesse que de la philosophie, puisqu'il va au-delà de ce que la philosophie peut offrir. Ce fut là une erreur heureuse et un pieux mensonge à l'attention des conjoints, car un parfait amour garantit de la sorte l'intégrité de l'union conjugale, et cela plus par l'innocence de l'âme que par la chasteté des corps. », idem.
} 
mariage : elle est celle de l'amitié pure et désintéressée, dégagée de toute convoitise. Par contraste, cette «concupiscentia» revient de manière insistante dans le portrait des mauvaises épouses. L'appétit, qui vise en l'autre la satisfaction de désirs charnels ou matériels, de la luxure ou du luxe, est condamné, qu'il se manifeste dans le mariage ou en dehors de lui. L'abbesse du Paraclet oppose moins les noms d'épouse et de concubine qu'elle ne déplace les frontières des concepts traditionnels pour faire passer la ligne de partage non plus entre la prostituée et l'épouse, mais au sein de ces deux catégories, entre des épouses et des prostituées «saintes » car désintéressées et douées de la «pudeur de l'âme », et des femmes dont les amours vénales n'ont de cette chasteté véritable que le simulacre, la «continence corporelle». Réciproquement, la «sainteté» qui est finalement reconnue à l'union entre époux par les deux occurrences de « sanctus » et celle de «beatus » ne lui est pas propre. Ce qui sanctifie l'amitié au sein du couple n'est pas, comme dans la doctrine officielle de l'Église, la conclusion du mariage, mais la pureté de l'intention dans laquelle chacun se porte vers cette union. Une femme peut donc être «beata meretrix » aussi bien que «beata sponsa ». Toutes deux recevront en retour non un loyer, «merces », mais une reconnaissance gratuitement octroyée, la «gratia», dont la «sainteté » peut apparaître à travers l'analogie possible avec la grâce divine.

Par là, l'éthique de l'intention élaborée par l'abbesse du Paraclet ${ }^{17}$ rejoint la condamnation du mariage par la jeune Héloïse rapportée par l'Historia :

17 Il aurait sans doute été plus exact de parler d'une application de l'éthique de l'intention au couple. Cette formule renvoie à l'idée défendue par Peter DRONKE qu'Héloïse n'est pas seulement la disciple d'Abélard, appliquant docilement ses idées, mais qu'elle a pu participer activement à l'élaboration de certaines notions. Le Scito te ipsum (Ethique) en particulier serait le fruit d'une réflexion commune, et non l'expression de la pensée du seul Abélard. Cf. Intellectuals and Poets in Medieval 
sibi carius existeret, michique honestius amicam dici quam uxorem ut me ei sola gratia conservaret, non vis aliqua vinculi nuptialis constringeret. ${ }^{18}$

D'après cette phrase, la notion d'obligation réciproque, qui fonde la définition du mariage chez les canonistes, est ce que rejette Héloïse. Elle rejoint en cela les condamnations courtoises du mariage comme contraire à l'existence même du désir. La reprise de cet argument quinze ans plus tard montre que, si Héloïse est vraisemblablement partie d'une conception courtoise dans son refus de l'arrangement imaginé par Abélard pour apaiser Fulbert et son lignage, ce qu'elle rejette au fond est la vénalité dont ce contrat entache son amour pour Abélard. L'opposition entre deux formes de mariage recouperait ainsi la distinction lexicale entre «matrimonii », systématiquement associé à «federa » ou à « honore » ${ }^{19}$, et « conjugatis ». Matrimonium désignerait le mariage dans sa conception ancienne, liée aux alliances entre familles, nécessairement intéressées, alors que conjugium renverrait plutôt aux réflexions des religieux du XII ${ }^{\mathrm{e}}$ siècle, qui cherchent à fonder l'union conjugale sur la dilectio que les époux doivent éprouver l'un pour l'autre ${ }^{20}$. C'est le matrimonium qui est condamné par Héloïse : c'est

Europe, Rome, Storia e Letteratura, 1992, p. 295-322 et p. 323-342 ; Women Writers of the Middle Ages, Cambridge, Cambridge University Press, 1984, p. 107-143.

${ }^{18}$ Historia Calamitatum, éd. cit., p. 17. Il ne semble pas avoir été remarqué jusqu'à présent que cette phrase, sur laquelle se conclut le plaidoyer d'Héloïse proprement dit dans l'Historia, n'est pas traduite par Jean de Meun, alors même qu'il se fonde sur cette idée pour donner à Héloïse une valeur exemplaire dans le Roman de la Rose. Voici la traduction : «il lui serait plus agréable, et à moi bien plus convenable, qu'on l'appelât plutôt ma maîtresse, et que seule la liberté de mon choix, et non un devoir quelconque ou la contrainte des chaînes nuptiales, me gardât auprès d'elle. », éd. cit., p. 73.

${ }^{19}$ Significativement, matrimonium n'est employé que dans ce passage. Dans ses autres lettres, Héloïse recourt à conjugio ou nuptiae, aux connotations plus positives. Nuptiae en particulier est dans la tradition chrétienne le terme privilégié pour évoquer les noces spirituelles. Ce pourrait être un argument supplémentaire en faveur du caractère relatif de la condamnation du mariage, qui n'est pas forcément considéré par Héloïse comme mauvais en soi, mais comme indifférent, seule comptant l'intention dans laquelle il est conclu. En somme, il serait une concession superflue à la société, qui introduit des obligations légales où seul l'amour peut garantir l'authenticité d'une union.

${ }^{20}$ Cf. Dom Jean LeCLERCQ, Le Mariage vu par les moines au XII siècle, Paris, Éditions du Cerf, 1983 (édition originale New York, The Seabury Press, 1982). 
parce que son mariage avec Abélard était conçu comme un matrimonium, un honneur pour la lignée ${ }^{21}$, et non comme un conjugium, qu'elle l'a refusé.

La variation dans la disposition des adjectifs attribués à « uxoris nomen » et «amice vocabulum » d'une formule à l'autre, irait dans le même sens d'un refus de la dimension officielle du matrimonium. Dans l'Historia, les deux adjectifs sont communément appliqués à « amicam ». La distinction «sibi carius [..] michique honestius » s'inscrit tout simplement dans la dynamique de l'argumentation qui précède : elle renvoie à la gloire du clerc, digne d'Abélard, donc chère à Héloïse. Dans la première reformulation de la lettre II, la distinction se transforme en opposition, «dulcius » s'appliquant désormais à « amice » et « sanctius ac validius » à « uxoris ». Les deux adjectifs, auparavant communs à un seul nom malgré la distinction de deux points de vue, deviennent des qualités antagonistes, appliquées à deux réalités séparées. Dans la dernière formule, « karius michi et dignius » sont de nouveau conjoints dans une application commune à «meretrix », et fusionnés dans un seul point de vue, celui d'Héloïse. Si «dulcius » et «carius » semblent appartenir à un même paradigme lexical, l'autre pôle est représenté par des adjectifs porteurs de sèmes différents. La seconde formule en particulier, propose un doublet, « sanctius ac validius ». Dans les deux autres figure uniquement un terme simple, «honestius » ou «dignius », qui ont des valeurs proches : tous deux peuvent se référer aussi bien aux codes sociaux, extérieurs, qu'à un code moral, intérieur. Au contraire, «validius » me paraît renvoyer à la force de la loi, à la validité socialement reconnue au mariage. Dans la

${ }^{21}$ Elle le dit explicitement dans la lettre IV, éd. cit., p. 64 : « et me pariter et totum genus meum sublimaveras », "tu avais déjà assez fait en t'abaissant pour moi, m'ennoblissant, ainsi que tout mon lignage» (je souligne). Il y a donc, dans le mariage avec Abélard, la même recherche d'honneur et de gloire que dans le fait d'épouser un empereur. 
mesure où c'est précisément lors du déplacement sur «uxor» que « validius » est introduit en doublet, cela confirmerait que la condamnation porte effectivement sur le matrimonium comme institution sociale, et que c'est l'introduction d'une dimension légale, extérieure, qui porte atteinte pour Héloïse à la valeur morale.

L'emploi de «sanctius » appelle de son côté une autre remarque. Dans les salutations, Héloïse hésite, pour définir son ethos d'épistolière, entre plusieurs qualifications, «ancilla sua, immo filia; ipsius uxor, immo soror ». Or au début de la lettre, lorsqu'elle parle au nom de l'ensemble des religieuses, elle reprend successivement les images de la servante, avec «ancillulas», et de la fille, avec «filiabus». Ces deux identités coïncideraient donc avec la charge qu'elle exerce au sein du Paraclet, où elle se considère moins comme abbesse que comme une moniale parmi les autres, et à ce titre une fille spirituelle et une servante du fondateur du monastère, Abélard. Les salutations forment ainsi une sorte de thème initial, à partir duquel l'ensemble de la lettre se développe à la façon d'une variation ou d'un commentaire, comme certaines chansons à partir de leur premier vers. Mais si les trois premiers termes sont repris successivement, jamais dans cette lettre, ni d'ailleurs dans les suivantes, Héloïse ne se désigne comme la «sœur» d'Abélard. Lui, au contraire, reprend uniquement ce dernier titre dans ses salutations, et en fait son vocatif de prédilection pour interpeller Héloïse. Or dans la lettre d'Héloïse, « soror », introduit par « immo », et «meretrix » ont tous deux un statut analogue par rapport au terme qu'ils sont censés remplacer, «uxor». Serait-il possible dans ces conditions que «meretrix» se substitue moins à «uxor» qu'à «soror $»^{22}$ ? La provocation qu'il y aurait à choisir comme titre définitif

\footnotetext{
${ }^{22}$ Un titre «plus saint » que les autres, employé par les membres de l'Église entre eux. En dehors de ses lettres à Abélard, Héloïse se qualifie de « soror», comme le montre sa lettre à Pierre le Vénérable (Cf. The Letters of Peter the Venerable, éd. cit., vol. 1,
} 
dans sa relation à Abélard «meretrix » n'en paraît que plus grande : c'est un retour aux origines de leur amour, un refus non seulement du premier ordre que lui a donné Abélard, mais du second, non seulement du mariage, mais surtout de l'entrée dans les ordres ${ }^{23}$. À partir du moment où elle ne parle plus au nom de l'ensemble des moniales du Paraclet mais en son nom propre, comme individu, elle refuse de s'adresser à Abélard autrement que comme à son amant.

Abélard répond «dilectissime sorori in Christo ». Le choix de ne retenir que «sorori » a dans une telle perspective valeur d'exhortation et d'encouragement à accepter comme sien ce statut. L'ajout de « dilectissime », qui est le vocatif favori d'Héloïse lorsqu'elle l'apostrophe dans la lettre II, irait en ce sens : il souligne que le passage dans les ordres n'implique aucunement une diminution de l'amitié entre eux, mais plutôt un accroissement. C'est ce qu'explicite la première apostrophe de cette lettre, «Soror in seculo quondam cara, nunc in Christo karissima $»^{24}$. Le parallèle met en valeur l'introduction en tiers du Christ, qui est l'innovation majeure d'Abélard. Il retravaille les salutations d'Héloïse en opérant à la fois un choix, celui de «soror », et un déplacement, du siècle au cloître. D'une certaine façon, cette introduction du Christ en tiers peut donc être une manière d'indiquer à Héloïse la seule voie possible pour atteindre le dernier terme de ses salutations et se l'approprier.

p. 400-401). C'est donc par rapport à Abélard, et à lui seul, qu'elle refuse d'être « sa sœur dans le Christ » comme il le voudrait.

${ }^{23}$ Ces remarques s'appliquent à la lettre II uniquement, et n'engagent aucun jugement sur la réalité ou non de sa « conversion » dans la suite de la correspondance.

${ }^{24}$ Dans les envois ultérieurs d'Abélard (cf. note 1), cette expression devient une formule de salutations. La relation d'Abélard à Héloïse se définit par la dynamique qui l'a forgée. Alors que dans les apostrophes d'Héloïse, le superlatif règne en maitre, traduisant l'intensité d'un sentiment qui ne se dément jamais, Abélard introduit une gradation. Le passage de «cara» à «carissima» matérialise ainsi le poids de la conversion dans son identité et la supériorité qu'il accorde à l'amour sacré sur l'amour profane. La conversion fait partie intégrante de l'amour d'Abélard, car elle seule a transformé la libido en véritable dilectio. De fait, Abélard ne s'adresse jamais à Héloïse comme amante, mais toujours comme « uxor, immo soror». 
Elle est aussi une invitation à remplacer la correspondance par la prière, suggestion appuyée, non par hasard, sur le statut d'épouse d'Héloïse. Aux inquiétudes d'Héloïse, qui redoute la mort de son amant, Abélard répond par une invitation à prier pour son salut. Après un éloge de la prière en général, il en vient à l'efficacité particulière des prières féminines, en s'appuyant sur des exemples bibliques qui mettent en valeur des liens affectifs et familiaux entre les orantes et ceux qu'elles ont sauvés. Se concentrant ensuite sur la seule Héloïse, il souligne que ses prières doivent être d'autant plus ardentes et seront d'autant plus entendues qu'elle est son épouse, ce qui les rend «plus justes ». La lettre s'achève sur une modification des prières faites par les sœurs pour Abélard ${ }^{25}$. Or les termes par lesquels il introduit cette modification,

Nostis, dilectissima, quantum karitatis affectum presentie mee conventus olim vester in oratione solitus sit exhibere. [...] Nunc autem absenti michi, tanto amplius orationum vestrarum opus est suffragio, quanto majoris anxietate periculi constringor $[\ldots]^{26}$

reprennent un topos de la rhétorique épistolaire, l'opposition de la présence et de l'absence. Abélard semble vouloir substituer à la correspondance, rituel de l'amitié profane, la prière comme rituel de l'amitié chrétienne où la figure divine s'interpose comme lieu d'union des âmes. La succession de «dilectissima» et d'une référence à la charité chrétienne, «karitatis affectum », va dans le même sens. Le topos épistolaire et l'apostrophe favorite d'Héloïse ${ }^{27}$ sont insérés dans un contexte chrétien qui les fait

\footnotetext{
${ }^{25}$ La lettre V s'achève également sur une prière écrite par Abélard pour Héloïse. Cette parenté de structure suffirait à démontrer l'importance du changement de genre aux yeux d'Abélard.

${ }^{26}$ Lettre III, éd. cit. p. 58-59. Voici la traduction : « Je n'ai pas besoin, ma bien-aimée, de te rappeler combien la charité t'a poussée, toi et ton couvent, à prier pour moi lorsque j'étais parmi vous, ni quels furent les effets de votre dévotion. [...] Mais maintenant que je suis loin de vous, le secours de vos prières m'est d'autant plus nécessaire que l'angoisse de mes périls va sans cesse augmentant. », éd. cit., p. 165.

27 « Dilectissime » revient trois fois, « karisssime » une, et « unice » deux fois.
} 
glisser vers une réalité autre. Abélard favorise ainsi une transition sans rupture vers le nouvel état qu'il souhaite voir adopter par Héloïse. Où elle voulait «des lettres et des chansons », comme dans l'amour profane mais transposées sur un thème chrétien, il souligne que la prière est la seule véritable chanson chrétienne, et que le changement de contenu ne peut que s'accompagner d'un changement de forme. Celui-ci implique également une reconfiguration de la relation, par l'introduction d'un tiers, Dieu, qui est le destinataire du nouveau discours, dans lequel s'unissent les voix. Au dialogue, se substitue le chœur; à l'amante, l'épouse et la sœur, deux figures privilégiées de la femme au psautier, qui bénéficient toutes deux d'un statut sacré ${ }^{28}$.

Par rapport à la chanson, qui incarne l'univers poétique profane, la position de la lettre est plus ambiguë. De fait, elle n'est pas l'objet d'une exclusion, mais plutôt d'une conversion ${ }^{29}$, accompagnée d'une restriction de son emploi. Dans la sphère profane, l'amante peut être épistolière, l'épouse moins aisément : la position la plus commune est de lui accorder le droit de recevoir des lettres, non d'en envoyer ${ }^{30}$. Un déséquilibre s'introduit au sein du couple dans l'emploi de l'écriture, peu favorable à la correspondance. De même, la religieuse semble plutôt faite pour recevoir des lettres de direction et pour prier que pour écrire. Les justifications pratiques du monde profane se trouvent remplacées par d'autres, symboliques, comme le fait que les épîtres bibliques représentent la parole de Dieu adressée aux fidèles à travers l'apôtre, qui est son représentant sur

\footnotetext{
${ }^{28}$ Puisque le mariage sanctifie les relations entre l'homme et la femme.

${ }^{29}$ Au sens religieux du terme. De même, la conversion du chant, à partir de sa forme profane, la chanson, donne la prière.

${ }^{30}$ On peut penser par exemple aux considérations du CHEVALIER DE LA TOUR-LANDRY dans son Livre pour l'éducation de ses filles à la fin du chapitre 90, «De aprendre saigesce et clergie ». Cf. Le Livre du Chevalier de la Tour Landry pour l'enseignement de ses filles, publié d'après les manuscrits de Paris et de Londres par Anatole DE MonTAiglon, Paris, P. Jannet, 1854, réimpr. Kraus Reprint, 1982, p. 178.
} 
terre et son porte-parole ${ }^{31}$. Les lettres de direction d'Abélard, à la fois époux, magister et fondateur du monastère, deviennent la voix de Dieu, qui s'adresse à ses épouses à travers son serviteur ${ }^{32}$. Du côté de la femme, la lettre ne subsiste en principe que pour des problèmes techniques, des demandes ponctuelles. La dimension spirituelle, donc émotionnelle et affective, est renvoyée de préférence vers la prière, où les cœurs se mettent à l'unisson en un chœur dirigé vers Dieu. Il en ressort que la véritable forme de l'affection entre époux est la prière de l'épouse pour la conservation du mari, ou la lettre de direction de celui-ci : il n'y a pas de véritable correspondance exprimant la dilectio, ou du moins les correspondances n'ont pas pour but d'exprimer cette affection, qui s'y développe par surcroît. L'épouse, contrairement à l'amante, n'est pas épistolière, ne dialogue pas avec son époux pour parler d'amour : elle est orante, et entretient Dieu de son amour pour son époux. Avec celui-ci, elle dialoguera plutôt autour de problèmes liés au ménage, c'est-à-dire, encore une fois, autour d'une réalité tierce, sur laquelle se greffe l'expression de l'affection, qui se nourrit de cette réalité commune - amor in conjugio, amor in Christo. La lettre de dilectio reste réservée, comme forme d'expression littéraire, aux amants.

La représentation du couple est extrêmement différente chez Abélard et chez Héloïse, d'où le débat qui se poursuit tout au long des lettres suivantes pour définir la relation sur laquelle peuvent se fonder leurs

\footnotetext{
${ }^{31}$ Sur ce point, voir Alain BOUREAU, « La norme épistolaire, une invention médiévale », in Roger CHARTIER (dir.), La Correspondance. Les usages de la lettre au XIX siècle, Paris, Fayard, 1991, p. 127-157, en particulier les p. 129-136.

${ }^{32} \mathrm{Ce}$ sera la lettre $\mathrm{V}$, qui opère également un déplacement métaphorique de la figure de l'épouse : Héloïse n'y est plus l'épouse réelle d'Abélard, mais l'épouse du Christ, par un emploi analogique de ce statut. Les religieux utilisent en effet les relations familiales comme modèle métaphorique pour penser leur communauté, comme le montrent les noms de «frère » et de «sœur », celui de «père »donné à l'abbé et de «mère » à l'abbesse.
} 
échanges intellectuels. Cette relation se définit avant tout par la dilectio qui les unit. Mais alors que celle-ci renvoie, pour Héloïse, à l'amour profane qu'elle a toujours éprouvé pour Abélard, pour celui-ci au contraire, elle a supposé une métamorphose liée à une sacralisation, et la figure qui l'incarne sera plutôt l'épouse ou la sœur « in Christo ». Ce débat a le mérite de rappeler que le mariage est une des formes de réalisation possible de l'amitié désintéressée, y compris pour Héloïse. En revanche, cette dilectio conjugale se voit refuser l'accès à l'expression littéraire, que ce soit chez Héloïse, qui écrit comme amante et non comme épouse, ou chez Abélard, qui préférerait que, en épouse aimante, elle prie pour lui. Dans ce silence imposé au mariage, interdit d'expression poétique, se rejoignent l'idéologie courtoise, qui considère l'obligation conjugale comme contraire au désir d'où jaillit le chant, et l'idéologie cléricale, pour laquelle la fonction sacerdotale, dont relève la maîtrise de l'écrit, et la fonction reproductrice, dont relève le mariage, ont tendance à s'exclure mutuellement. De fait, si Abélard et Héloïse reviennent, quinze ans après les faits, sur la question du mariage et interrogent sa validité comme ethos épistolaire, ce n'est pas pour lui-même, mais à cause de sa valeur métaphorique et parce que le premier acte de soumission d'Héloïse, l'acceptation du mariage, renvoie au second, son entrée dans les ordres, qui en appellera, dans la lettre VI, un troisième, le silence imposé à certaines de ses pensées. 